\title{
Autoimmune thyroid disease and urticarial vasculitis: is there a significant association?
}

\author{
Ivan Cherrez-Ojeda ${ }^{1,2^{*}} \mathbb{D}$, Emanuel Vanegas ${ }^{1,2}$, Valeria L. Mata², Miguel Felix², German D. Ramon ${ }^{3}$, \\ Sofia Cherrez ${ }^{2,4}$ and Annia Cherrez $z^{2,5}$
}

\begin{abstract}
Little is known about the association of urticarial vasculitis (UV) with thyroid autoimmunity. The latter has been mostly described in the setting of patients with chronic spontaneous urticaria (CSU). In this letter to the editor, we compare UV and CSU through retrospective analyses, which reveal that $41.7 \%$ patients with UV presented antithyroperoxidase (anti-TPO) and/or antithyroglobulin antibody (ATA) above the reference range, while only $4 \%$ patients with CSU had these antibodies elevated. There is a moderately strong association assessed by the Phi coefficient $(\varphi=0.420, p=0.004)$. Further research is needed to appropriately address the relationship between $U V$ and thyroid autoimmunity and explore any potential underlying pathophysiological process between both diseases.
\end{abstract}

Keywords: Urticarial vasculitis, Thyroid autoimmunity, Chronic spontaneous urticaria, Autoantibody, Immune response

Urticarial vasculitis (UV) is identified as a clinicopathologic entity that involves clinical features of urticaria and histopathologic findings compatible with a cutaneous leukocytoclastic vasculitis of small vessels with fibrinoid deposits. UV represents a spectrum of diseases that differ in severity, ranging from an urticaria with minimal vasculitis to a systemic disease that can lead to serious organ-specific complications. Certainly, urticarial vasculitis is an underdiagnosed disease, to the extent that its incidence may vary from 3 to $20 \%$ [1]. This is due to the lack of a consensus in medical literature upon a disease that manifests diversely, with a definite diagnosis that relies on a high-tech procedure such as biopsy.

Several findings may facilitate its diagnosis, such as abnormal acute phase reactants, hypocomplementemia, positive antinuclear antibodies (ANA) and other laboratory tests that have been extensively described, as well as common associations with other clinical conditions such as systemic lupus erythematosus

\footnotetext{
*Correspondence: ivancherrez@gmail.com

1 Universidad Espíritu Santo, Km. 2.5 vía La Puntilla, Samborondón Zip code: 0901-952, Ecuador

Full list of author information is available at the end of the article
}

(SLE), lupus-like disease and Sjogren's Syndrome [1, 2]. However, little is known of how UV associates with thyroid autoimmunity. The latter has been mostly described in the setting of patients with chronic spontaneous urticaria (CSU), with evidence suggesting that CSU can improve in response to treatment with levothyroxine or other thyroid drugs [3]. In this report we discuss the potential implication of screening UV patients for abnormally elevated IgG anti-thyroid Abs (AAbs), as this feature has been described as a predictor of hypothyroidism in euthyroid patients [4].

We carried out a retrospective analysis from 2005 to 2016 involving 98 patients diagnosed with either chronic spontaneous urticaria or urticarial vasculitis at Respiralab Urticaria Center, Guayaquil-Ecuador. Demographic and clinical variables such as age, sex, years with the disease, type of urticaria, thyroid function tests and antithyroid antibodies were collected using medical records from the institution. The diagnostic definitions used for chronic spontaneous urticaria and urticarial vasculitis are summarized in Table 1 [5-8]. All patients with clinical features suggestive of urticarial vasculitis were confirmed by biopsy. Thyroid function tests (T3, T4, TSH) were categorized into normal, below normal or above normal, 
Table 1 Definition of chronic spontaneous urticaria and urticarial vasculitis

\section{Chronic spontaneous urticaria \\ Spontaneous development of wheals, angioedema, or both for \\ $>6$ weeks. Wheals have the following characteristics \\ 1. Central swelling surrounded by reflex erythema. \\ 2. Itching, sometimes burning sensation \\ 3. Skin returning to normal appearance within $30 \mathrm{~min}$ to $24 \mathrm{~h}$ \\ Urticarial vasculitis \\ Clinical manifestations of urticaria characterized by wheals that are painful, burning, and tender in sensation. Lesions tend to last $>24 \mathrm{~h}$ and are associated with faint residual hyperpigmentation. Biopsy is needed to confirm the diagnosis, with the following findings \\ 1. Inflammatory injury to capillaries and postcapillary venules \\ 2. Leukocytoclasis with fibrinoid deposits as signs of direct vessel damage}

References: [5-8]

while AAbs (antithyroperoxidase, antithyroglobulin antibodies) where dichotomized as absent or present.

Descriptive analyses (frequency, percentage, standard deviation) were carried out for demographic and clinical variables. The Chi square test was applied to analyze the fit of distribution and to measure association with the Phi coefficient between the diagnosis and the presence/ absence of AAbs. Crude analysis with binomial logistic regressions between diagnosis and antithyroid antibodies were performed. All statistical analyses were carried out using SPSS v24.0 (IBM, Armonk, NY, USA). $P<0.05$ was considered significant.

Descriptive analyses from the 98 patients involved showed that $25.5 \%(n=25)$ were males and $74.5 \%$ $(n=73)$ were females. The mean age of the sample studied was 38.12 years (SD 15.97), and the mean length of the disease since diagnosis was 1.49 years (SD 2.19). Concerning the disease, $87.8 \%(n=86)$ patients were diagnosed with chronic spontaneous urticaria and $12.2 \%$ $(\mathrm{n}=12)$ with urticarial vasculitis (Fig. 1$)$.

From 12 patients with urticarial vasculitis, $41.7 \%$ $(\mathrm{n}=5)$ presented antithyroperoxidase (anti-TPO) and/ or antithyroglobulin antibody (ATA) above the reference range $(\mathrm{p}=0.564)$, while only $4 \%(\mathrm{n}=4)$ out of the 86 patients with CSU had these AAbs elevated $(p=0.000)$. The observed frequency for patients with AAbs above normal with urticarial vasculitis was greater than the expected value, while for CSU patients it was less than expected, with a moderately strong association assessed by Phi coefficient $(\phi=0.420, p=0.004)$. Patients with UV were 14.64 times more likely to have abnormally elevated levels of AAbs than CSU patients $(p=0.001)$. Regarding complement $66.6 \%(n=8)$ of patients had low levels (defined by low $\mathrm{C} 3$ and $\mathrm{C} 4)$, and $8.3 \%(\mathrm{n}=1)$ had normal levels. Unfortunately, $25 \%(n=3)$ of patients did not have complement measurements. There were no UV patients presenting with associated SLE, or related collagen disorder.

With respect to thyroid profile on individuals with elevated AAbs, we found that $75 \%(n=3)$ patients with CSU were euthyroid, while $25 \%(n=1)$ were compatible with subclinical hyperthyroidism. On the other hand, $60 \%(n=3)$ cases diagnosed with UV were euthyroid, while $20 \%(n=1)$ were compatible with subclinical hypothyroidism and another $20 \%(\mathrm{n}=1)$ had T3 levels above normal with T4 and TSH in the reference range.

Annual reassessment of thyroid function and AAbs levels in UV patients may be recommended, since levels of AAbs have shown to predict the future development of hypothyroidism in an euthyroid general population [4]. In our sample, all patients with elevated AAbs were euthyroid, except for three patients, of which two were compatible with subclinical hypothyroidism. Currently, it is known that the presence of AAbs in the patient

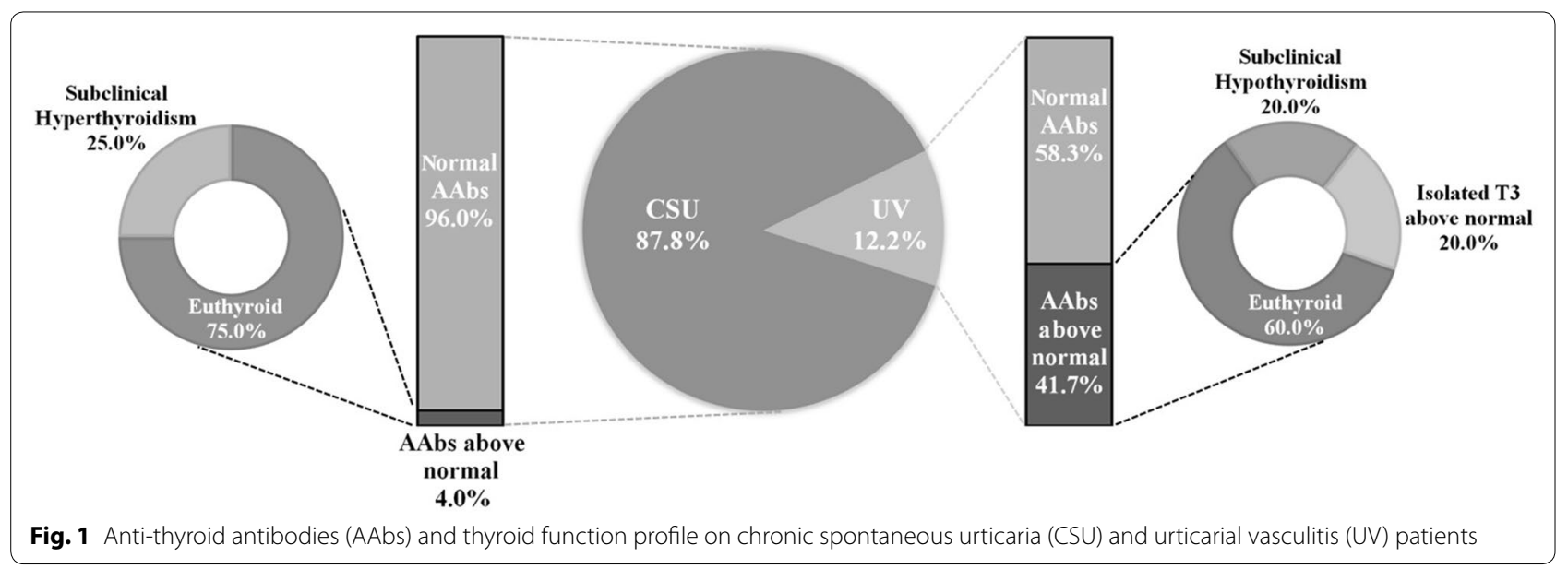


with CSU does not associate with abnormal thyroid function, since most patients are usually euthyroid [9]. However, in a minority of patients with Hashimoto's thyroiditis, administration of levothyroxine may improve chronic urticaria symptoms, hypothetically highlighting a common pathogenesis between such entities [10]. It is not known if treating thyroid disease in UV patients might resolve urticaria symptoms in some degree, a point we consider of interest for future research.

As relevant limitations to our study we must mention the small sample size and retrospective nature. Nevertheless, it would be interesting to develop new research to appropriately address the relationship between UV and thyroid autoimmunity, to explore if there is an underlying pathophysiological process between both diseases, and to determine if early treatment of thyroid disease is recommended. Only this data would, in clinical practice, support the screening of AAbs in UV patients.

\section{Abbreviations}

UV: urticarial vasculitis; ANA: antinuclear antibodies; SLE: systemic lupus erythematosus; CSU: chronic spontaneous urticaria; AABs: anti-thyroid antibodies; anti-TPO: antithyroperoxidase; ATA: antithyroglobulin antibody.

\section{Authors' contributions}

Authors have made substantial contributions to conception and design, acquisition, analysis and interpretation of data, have been involved in drafting the manuscript or revising it critically for important intellectual content, and given final approval of the version to be published. ICO, VLM, GDR formulated the study question, EV, MF wrote the manuscript. SC, AC revised the final comments. All authors read and approved the final manuscript.

\section{Author details}

1 Universidad Espíritu Santo, Km. 2.5 vía La Puntilla, Samborondón Zip code: 0901-952, Ecuador. ${ }^{2}$ Respiralab, Respiralab Research Group, Guayaquil, Ecuador. ${ }^{3}$ Allergy Section, Hospital Italiano Regional del Sur, Bahía Blanca, Argentina. ${ }^{4}$ University of Heidelberg, Heidelberg, Germany. ${ }^{5}$ Clinic and Policlinic for Dermatology and Venereology, University Medical Center Rostock, Rostock, Germany.

\section{Acknowledgements}

Not applicable.

\section{Competing interests}

The authors declare that they have no competing interests.

\section{Availability of data and materials}

The datasets used and/or analysed during the current study are available from the corresponding author on reasonable request.
Consent for publication

Not applicable.

\section{Ethics approval and consent to participate}

This work has been approved under the consent of the Hospital-Clinica Kennedy's ethics committee, in accordance to the principles established by the declaration of Helsinski.

\section{Funding}

No support from any organization for the submitted work has been provided.

\section{Publisher's Note}

Springer Nature remains neutral with regard to jurisdictional claims in published maps and institutional affiliations.

Received: 12 April 2018 Accepted: 4 April 2019

Published online: 18 April 2019

\section{References}

1. Venzor J, Lee WL, Huston DP. Urticarial vasculitis. Clin Rev Allergy Immunol. 2002;23(2):201-16.

2. Asherson RA, D'Cruz D, Stephens CJ, McKee PH, Hughes GR. Urticarial vasculitis in a connective tissue disease clinic: patterns, presentations, and treatment. Semin Arthritis Rheum. 1991;20(5):285-96.

3. Nuzzo V, Tauchmanova L, Colasanti P, Zuccoli A, Colao A. Idiopathic chronic urticaria and thyroid autoimmunity: experience of a single center. Dermato-endocrinology. 2011;3(4):255-8.

4. Roos A, Links TP, Berg LT, Gans RO, Wolffenbuttel BH, Bakker SJ. Thyroid peroxidase antibodies, levels of thyroid stimulating hormone and development of hypothyroidism in euthyroid subjects. Eur I Int Med. 2010;21(6):555-9.

5. Zuberbier T, Aberer W, Asero R, Abdul Latiff AH, Baker D, Ballmer-Weber B, Bernstein JA, Bindslev-Jensen C, Brzoza Z, Buense Bedrikow R, et al. The EAACI/GA(2)LEN/EDF/WAO guideline for the definition, classification, diagnosis and management of urticaria. Allergy. 2018;73(7):1393-414.

6. Warin RP. Urticarial vasculitis. British medical journal (Clinical research ed). 1983;286(6382):1919-20.

7. Black AK, Lawlor F, Greaves MW. Consensus meeting on the definition of physical urticarias and urticarial vasculitis. Clin Exp Dermatol. 1996;21(6):424-6.

8. Jones RR, Bhogal B, Dash A, Schifferli J. Urticaria and vasculitis: a continuum of histological and immunopathological changes. $\mathrm{Br} J$ Dermatol. 1983;108(6):695-703.

9. Kaplan AP, Finn A. Autoimmunity and the etiology of chronic urticaria. J All Clin Immunol. 1999;4:286-92.

10. Kim DH, Sung NH, Lee AY. Effect of levothyroxine treatment on clinical symptoms in hypothyroid patients with chronic urticaria and thyroid autoimmunity. Ann Dermatol. 2016;28(2):199-204.

\footnotetext{
Ready to submit your research? Choose BMC and benefit from:

- fast, convenient online submission

- thorough peer review by experienced researchers in your field

- rapid publication on acceptance

- support for research data, including large and complex data types

- gold Open Access which fosters wider collaboration and increased citations

- maximum visibility for your research: over 100M website views per year
}

At BMC, research is always in progress.

Learn more biomedcentral.com/submissions 